\title{
Design and Evaluation of the Unmanned Technology Research Center Exoskeleton Implementing the Precedence Walking Assistance Mechanism
}

\author{
Dowan Cha**, Sung Nam Oh*, Hee Hwan Lee*, Kyung-Soo Kim**, Kab Il Kim* \\ and Soohyun Kim ${ }^{\dagger}$
}

\begin{abstract}
Assistance of the operator's walking ability while carrying a load is a challenging area in lower limb exoskeletons. We implement an exoskeleton called the Unmanned Technology Research Center Exoskeleton (UTRCEXO), which enables the operator to walk with a load more comfortably. The UTRCEXO makes use of two types of DC motor to assist the hip and knee joints. The UTRCEXO detects the operator's walking intention including step initiation with insole-type FSRs faster without using any bio-signals and precedes the operator's step with a reference torque. It not only reduces interaction forces between the operator and the UTRCEXO, but also allows the operator to walk with a load more comfortably. In this paper, we present the UTRCEXO implementing the walking assistance mechanism with interaction force reduction during walking.
\end{abstract}

Keywords: The lower limb exoskeleton, Walking intention, Interaction force

\section{Introduction}

A lower limb exoskeleton is an active mechanical device that assists the operator in walking more comfortably with loads, or enables persons suffering from paraplegia to walk [1-2]. It can be described as a walking assistive exoskeleton and an orthotic exoskeleton, respectively. There are six important technologies for the lower limb exoskeleton: actuator technology, mechanism technology, control technology, sensor technology, detection of human walking intention technology, and human-robot interface technology [3-4]. In particular, the detection of human walking intention has been an important challenge in developing a lower limb exoskeleton. Walking assistive exoskeletons attempt to detect human walking intention including step initiation as soon as possible because a faster detection of step initiation initiates first step of the exoskeleton faster, but also because it allows the operator to walk more comfortably [5-8]. There are two types of approach to detect walking intention including step initiation in the walking assistive exoskeleton: a precedence walking assistance mechanism and a shadow walking assistance mechanism [9].

The precedence walking assistance mechanism detects the walking intention including step initiation of the operator prior to visible movements of the operator by

\footnotetext{
$\dagger$ Corresponding Author: Dept. of Mechanical Engineering, KAIST, Korea. (soohyun@kaist.ac.kr)

* Dept. of Electrical Engineering, Myong Ji University, Korea (kkl@mju.ac.kr)

** Dept. of Mechanical Engineering, KAIST, Korea.

Received: May 20, 2014; Accepted: June 24, 2015
}

using bio-signals. The robot suit Hybrid Assistive Limb (HAL) makes use of such bio-electrical signals, including ElectroMyoGraphy (EMG), and Floor Reaction Force (FRF), to detect the walking intention including step initiation of the operator before the corresponding visible movements occur [10-11]. As a result, the HAL can not only initiate its step before the step of the operator, but also precede the steps of the operator more comfortably. However, the issues encountered with bio-signals, such as low reliability, and long calibration times for each person have limited further development of the precedence walking assistance mechanism. The HAL has DC motors at each hip and knee joint with a harmonic drive placed directly on the joints. Additionally, the HAL utilizes a number of sensors to operate the suit, including potentiometers, FRF sensors, a gyroscope, and accelerometer [12].

In contrast, the shadow walking assistance mechanism detects the walking intention including step initiation of the operator after the visible movements of the operator occur, such as the heel-off event or the toe-off event. The RoboKnee detects the walking intention based on both the center of pressure and the heel-off event, when the heel is lifted just before the front end of the foot leaves the ground. It does so by using two load cells installed in stiff-bottom bicycle shoes. The RoboKnee has a linear Series Elastic Actuator (SEA) connected between the knee and hip. The Roboknee amplifies power at the knee to assist the walking ability of the operator [13-14]. The Berkeley Lower Extremity Exoskeleton (BLEEX) detects the walking intention including step initiation of the operator with toeoff events, when the toe leaves the ground, by using a foot switch installed in their shoes [15-16]. The BLEEX makes 
use of a number of sensors to operate itself, including encoders, linear accelerometers, force sensors, and an inclinometer [17].

These approaches are reliable because they detect walking intention, including step initiation, after visible movements related to walking have occurred. However, these approaches have stronger interaction forces between the operator and the walking assistive exoskeleton, requiring faster control schemes based on the good dynamics of the system. They also require more sensors in order to operate.

Meanwhile, orthotic exoskeletons must attempt to detect the walking intention of the operator including step initiation more accurately, because they are intended for paraplegic users who cannot provide any motion of the legs and have difficulty with stability [18-19]. The ReWalk detects walking intention of the operator including step initiation by using torso angles and buttons installed in forearm crutches [20]. The eLegs detects walking intention of the operator including step initiation by using arm motion with forearm crutches [21].

In this paper, we describe a walking assistive exoskeleton called the Unmanned Technology Research Center Exoskeleton (UTRCEXO). The UTRCEXO implements the precedence walking assistance mechanism for walking including step initiation without using bio-signals. We analyzed the human gait from a quiet standing position to continuous walking using force plates and motion capture devices. We implemented the precedence walking assistance mechanism in the UTRCEXO with the peak time approach and then evaluated its performance.

We provide details and specifications of the UTRCEXO in Section 2. We then discuss the method how to detect walking intention of the operator in Section 3. Next, we discuss the evaluation of the UTRCEXO in Section 4. Finally, in Section 5, we discuss the results and future work.

\section{Design}

Fig. 1 shows an image of the UTRCEXO. The UTRCEXO has two actuated degrees of freedom in each leg for hip flexion/extension and knee flexion/extension. Additionally, the UTRCEXO has two actuated degrees of freedom in each arm for shoulder flexion/extension and elbow flexion/extension. In total, the UTRCEXO has 8 actuators. The UTRCEXO provides hip and shoulder abduction/adduction without any actuator. Meanwhile, the UTRCEXO has a modular design with variants that include a lower limb exoskeleton without arms and a whole body exoskeleton with arms. The torso section of the UTRCEXO consists of a rigid link to match with the spine of the operator and a plate on which to hang a load. The UTRCEXO attaches to the operator at the waist, each thigh, each shank, and each foot by using straps.

Additionally, there are two shoulder straps with a bag for the load. Table 1 lists the specification of the UTRCEXO in

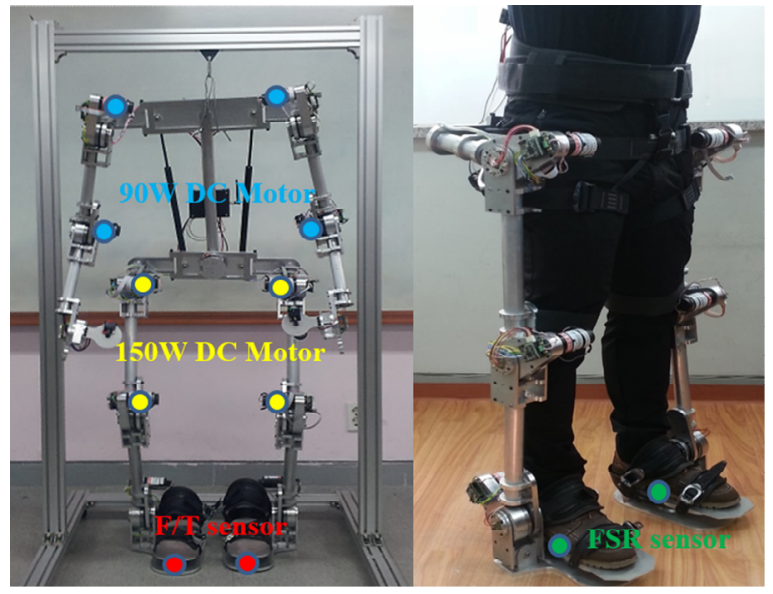

Fig. 1. Image of the UTRCXO (O: $90 \mathrm{~W}$ DC motor, O: $150 \mathrm{~W}$ DC motor, $\mathrm{O}$ F/T sensor, O: Insole-type FSRs)

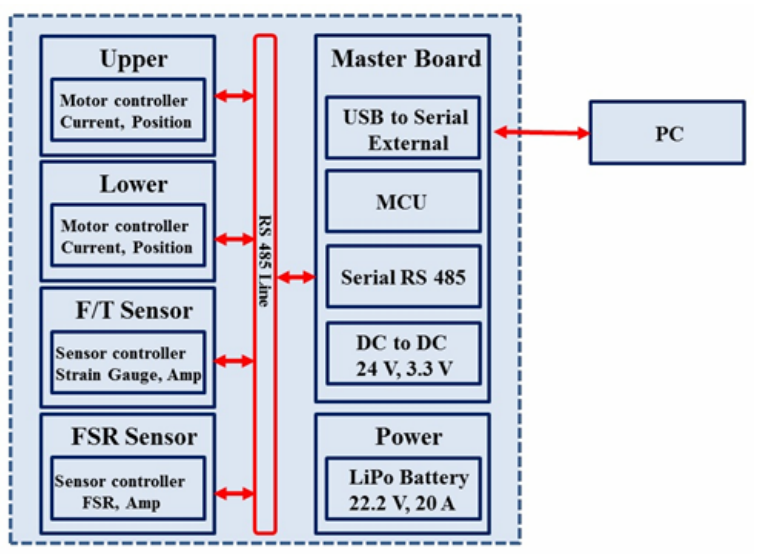

Fig. 2. System of the UTRCXO.

Table 1. Specification of the UTRCEXO

\begin{tabular}{c|c|c|c|c}
\hline \multirow{2}{*}{} & \multicolumn{2}{|c|}{ Higher Limb } & Lower Limb & \multirow{2}{*}{ Whole } \\
\cline { 2 - 4 } & Arms & Body & Legs & \\
\hline Length $(\mathrm{mm})$ & 700 & 750 & 900 & 1650 \\
\hline Weight $(\mathrm{Kg})$ & 8 & 3.5 & 18 & 29.5 \\
\hline D.O.F & \multicolumn{2}{|c|}{4} & 4 & 8 \\
\hline DC motor $(\mathrm{W})$ & \multicolumn{2}{|c|}{90} & 150 & \\
\hline Reduction ratio & $233: 1$ & $233: 1$ & \\
\hline
\end{tabular}

detail, and Fig. 2 shows the system of the UTRCEXO in detail. The main components of the UTRCEXO are introduced in the following subsections.

\subsection{Actuator}

The UTRCEXO has 8 actuators. Fig. 3 shows the actuator of the UTRCEXO. The actuator was developed as a whole body type actuator, including a reduction gear, a motor, a sensor, and a controller. The actuators are capable of performing both position control and torque control using position sensors and current sensors. The UTRCEXO has two types of DC motor: $90 \mathrm{~W}$ DC motors at each arm 


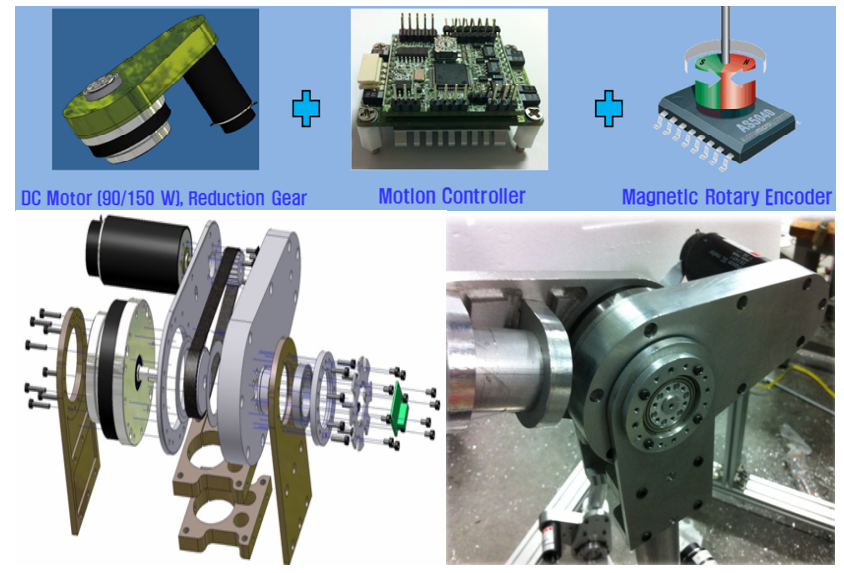

Fig. 3. Whole body type actuator. It consists of a reduction gear, a motor, a sensor, and a controller.

and $150 \mathrm{~W}$ DC motors at each leg, with a 233:1 harmonic drive gear reduction. The actuators have an absolute magnetic rotary encoder (MRE) to measure joint range of motion (ROM) of the UTRCEXO from reference position. The resolution of the MRE is 360 degrees/12 bits with 0.09 degrees of error. The motion controller makes use of a micro controller unit (STM 32F 103) based on a 32 bit ARM M3 core, and its power consumption is $300 \mathrm{~W}$ (24 $\mathrm{V}, 15 \mathrm{~A})$. The motion controller utilizes an RS 485 communication line with a $3 \mathrm{Mbps}$ and the sampling rate is $50 \mathrm{~Hz}$. A current sensor provides further feedback for the control system. The resolution of the current sensor is 12 bits. The current provides a measurement of torque, which is driven by the controller to a reference. The reference torque for each joint was calculated from an inverse dynamics model based on gait pattern analysis of subjects measured by force plates [22-23].

\subsection{Sensors}

We developed insole-type force sensing resistors (FSRs) to detect walking intention including step initiation of the operator [24]. The UTRCEXO has only a single twochannel FSR (FlexiForce 251bs) for each foot, with a sensor on the heel and the toe, and a MCU. Measured data are filtered using an 8 channel Op-amp filter with a cutoff frequency of $1 \mathrm{kHz}$. The force sensitivity range is from 0.1 $\mathrm{N}$ to $10 \mathrm{~N}$, and the pressure sensitivity range is from $1.5 \mathrm{psi}$ to $150 \mathrm{psi}$. The resolution of the insole-type FSRs is 12 bits. The sampling rate of the insole-type FSRs is $10 \mathrm{kHz}$. Additionally, we developed a 3 axis (X-Y-Z) F/T sensor, which is shown in Fig. 4. We utilized a binocular tunnel for the flexibility of a strain gauge. F/T sensors were installed in the footplates of the UTRCEXO and measure the interaction forces between the operator and the UTRCEXO. The range of force is from $100 \mathrm{~g}$ to $120 \mathrm{~kg}$ and the resolution of the $\mathrm{F} / \mathrm{T}$ sensor is $12 \mathrm{bit}$. The sampling rate of the $\mathrm{F} / \mathrm{T}$ sensor is $10 \mathrm{kHz}$ and the cutoff frequency is $100 \mathrm{~Hz}$.

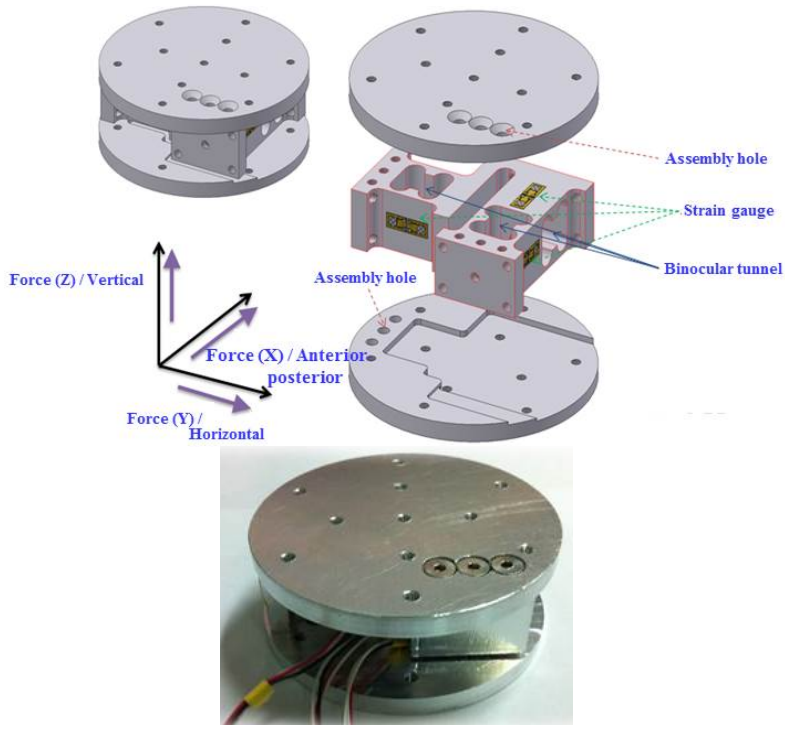

Fig. 4. Image of the F/T sensor.

\subsection{Control scheme}

The UTRCEXO detects step initiation of the operator using the peak time approach from the insole-type FSRs. The peak time approach detects step initiation of the operator with two particular ground reaction force events before visible movements occur, such as heel-off, knee flexion, and toe-off movement [25]. Once the UTRCEXO detects step initiation, the human intention detector of the UTRCEXO transmits each required joint torque as a reference torque to the motion controller using a RS485 communication line. These reference torques were calculated from an inverse dynamics model based on the gait pattern analysis of subjects with force plates [22]. The UTRCEXO starts to walk prior to the walking of the operator. During continuous walking, the UTRCEXO makes a gain selection with the walking intention of the operator based on a previous step velocity. With the gain selection, the UTRCEXO is still being controlled according to the walking intention of the operator. Additionally, the UTRCEXO detects step termination with the interaction force between the operator and the UTRCEXO, as measured by the F/T sensors installed in each foot-plate. Fig. 5 shows the flowchart of the control scheme. The sampling rate of the controller is $50 \mathrm{~Hz}$.

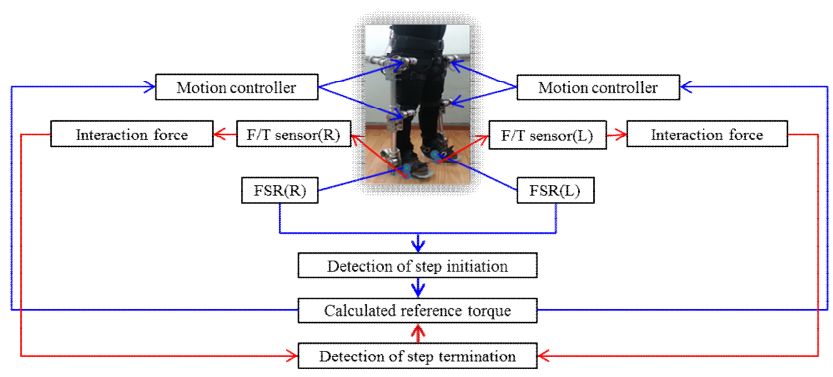

Fig. 5. Flowchart of the control scheme. 


\section{Detection of Walking Intention}

We developed an algorithm to detect the walking intention of the operator. We modeled the unit walking behaviors of the UTRCEXO as states of the UTRCEXO from Moore Automation for Robot Behavior (MARB) [2627]. A transition function determined step initiation and proper walking behaviors of the operator. MARB A is defined as:

$$
\mathrm{A}=\left\{\mathrm{Q}, \Sigma, \Omega, \delta, \lambda, \mathrm{q}_{0}\right\}
$$

For the set of conditions C, and the set of operations Op, the elements are defined as:

$$
\mathrm{Q}=\mathrm{Op} \times(\mathrm{C})^{*} \text { corresponds to the set of states }
$$

For $\mathrm{q} \in \mathrm{Q}: \mathrm{q}=\left(\mathrm{id}, \mathrm{op},\left(\mathrm{c}_{1}, \mathrm{id}_{1}\right), \ldots,\left(\mathrm{c}_{|\mathrm{q}|}, \mathrm{id}_{|\mathrm{q}|}\right)\right)$, $\mathrm{c}::=$ true $\mid$ false $\left|\left(\mathrm{c}_{\mathrm{k}}{ }^{\circ} \mathrm{c}_{\mathrm{j}}\right)\right| \mathrm{f}(\mathrm{v}) \in \mathrm{C}$

where

- id is the state's unique identifier

- Op is the set of operation

- $\mathrm{C}$ is the set of conditions of the transition

- the $\left(\mathrm{c}_{|\mathrm{q}|}, \mathrm{id}_{|\mathrm{q}|}\right)$ encode the outgoing transitions

- $|\mathrm{q}|$ denotes the number of conditions of state $\mathrm{q}$

- $\mathrm{c}$ is defined as condition

- $\circ$ is $\circ \in\{$ AND,OR

- $\mathrm{c}_{\mathrm{k}}, \mathrm{c}_{\mathrm{j}}$ are conditions

- $\mathrm{f}(\mathrm{v})$ is the function to judge that the inputs

For any element $\mathrm{x}$ of $\mathrm{A}$, the notation $\mathrm{x}^{\mathrm{q}}$ denotes that $\mathrm{x}$ belongs to state $\mathrm{q}$.

- The input alphabet $\Sigma=\mathrm{V}=(\mathrm{B})^{|\mathrm{H}|}$

- The output alphabet $\Omega=\mathrm{Op}$

- The transition function $\delta: \mathrm{Q} \times \mathrm{V} \rightarrow \mathrm{Q}$

$$
\begin{aligned}
& \delta(\mathrm{q}, \mathrm{v})= \\
& \left\{\begin{array}{l}
\mathrm{q}, \text { if } \exists\left(\mathrm{c}_{\mathrm{k}}, \text { id }_{\mathrm{k}}\right) \text { in } \mathrm{q}: \operatorname{id}_{\mathrm{k}} \text { and } \mathrm{E}\left[\left[\mathrm{c}_{\mathrm{k}}, \mathrm{v}\right]\right]=\text { true } \\
\mathrm{q}, \text { otherwise }
\end{array}\right.
\end{aligned}
$$

for $\mathrm{q} \in \mathrm{Q}, \mathrm{v} \in \mathrm{V}$.

- The output function $\lambda: \mathrm{Q} \rightarrow \mathrm{Op}: \lambda(\mathrm{q})=\mathrm{op}^{\mathrm{q}}=\left(\mathrm{cmd}^{\mathrm{q}}\right.$, $\operatorname{par}^{\mathrm{q}}$ ), for $\mathrm{q} \in \mathrm{Q}$.

- The initial state $\mathrm{q}_{0} \in \mathrm{Q}$.

In the UTRCEXO, the output range of the 4 FSR sensors and the $2 \mathrm{~F} / \mathrm{T}$ sensors is $\mathrm{B}=\{0, \ldots, 255\}$ and $\mathrm{H}=\left\{\mathrm{h}_{1}, . ., \mathrm{h}_{6}\right\}$. $\mathrm{v}=\left\{\mathrm{v}_{1}, . ., \mathrm{v}_{6}\right\} \in \mathrm{V}=\mathrm{B}^{6}$ refers to the acquired data from the FSRs and F/T sensors. If the condition is true, the transition occurs to operate the UTRCEXO with the output function.
We previously analyzed human gait patterns from step initiation to continuous walking, and proposed a new approach called the peak time approach to detect step initiation [25]. For the UTRCEXO, we defined the states from step initiation to step termination with the acquired data from FSRs and F/T sensors. It has a total of 6 states as follows:

- Equipment/Standing

- Step initiation

- First step

- Continuous walking

- Step termination

- Walking completion

Walking intention of the operator is detected with these states and the transition of these states. The UTRCEXO operates with an output function according to the state from the transition function. There are 3 commands including step initiation, continuous walking and step termination. Additionally, there are 2 parameters including right and left. The state of the UTRCEXO changes with the function described in (2). The transition function determines the next state from input alphabet and conditions. The input alphabets are values from FSRs and F/T sensors. The UTRCEXO detects step initiation using two particular events from FSRs with the peak time approach, and then walks continuously according to the intention of the operator. The UTRCEXO detects the intention of the operator for continuous walking and step termination with values from $\mathrm{F} / \mathrm{T}$ sensors. There are thresholds to determine the intention of the operator based on human gait analysis and trial/error.

\section{Evaluation}

The operators during the evaluation did not have postural problems and gave informed consent, approved by the Institutional Review Board of KAIST prior to participating in the evaluation. The operators' height (mean value) was $1.76 \mathrm{~m}$ and weight (mean value) was $73 \mathrm{~kg}$. The operators wore the UTRCEXO with insole-type FSRs installed in his shoes. They stood with both feet and, upon the instruction to walk, tried to step with their right foot first with their preferred step velocity. Then, they terminated their step when they wanted in limited space. Fig. 6 shows the sequence of the UTRCEXO implementing the precedence walking assistance mechanism. The step velocity of the UTRCEXO in this evaluation was $3.9 \mathrm{~km} / \mathrm{h}$. There was a $10 \mathrm{~kg}$ payload hung on the back plate. The UTRCEXO walked, including step initiation prior to the walking of the operator according to the intention of the operator. 


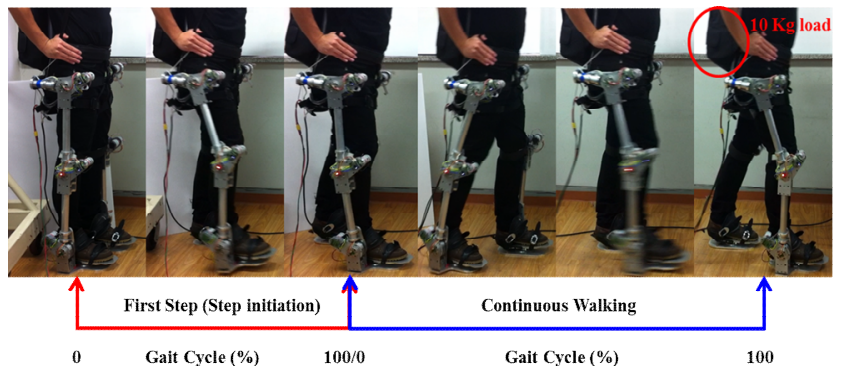

Fig. 6. Sequence of the UTRCEXO implementing the precedence walking assistance mechanism

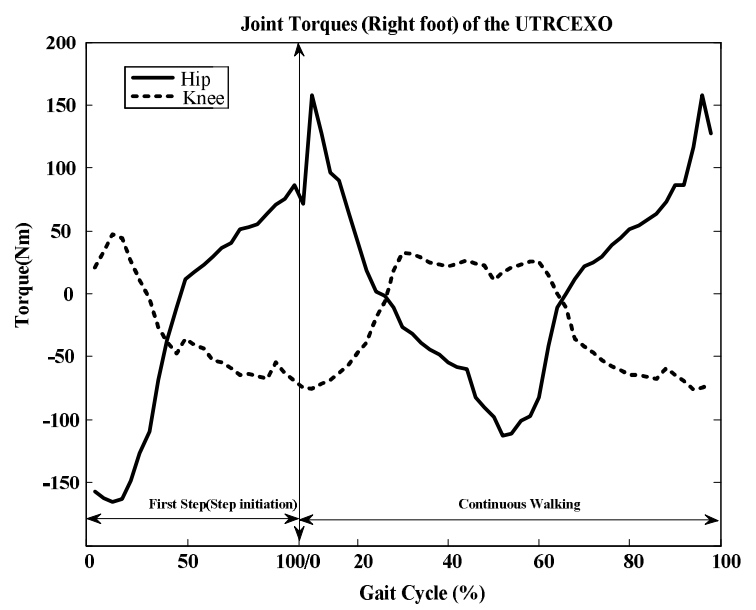

Fig. 7. Joint torques of the UTRCEXO for right foot

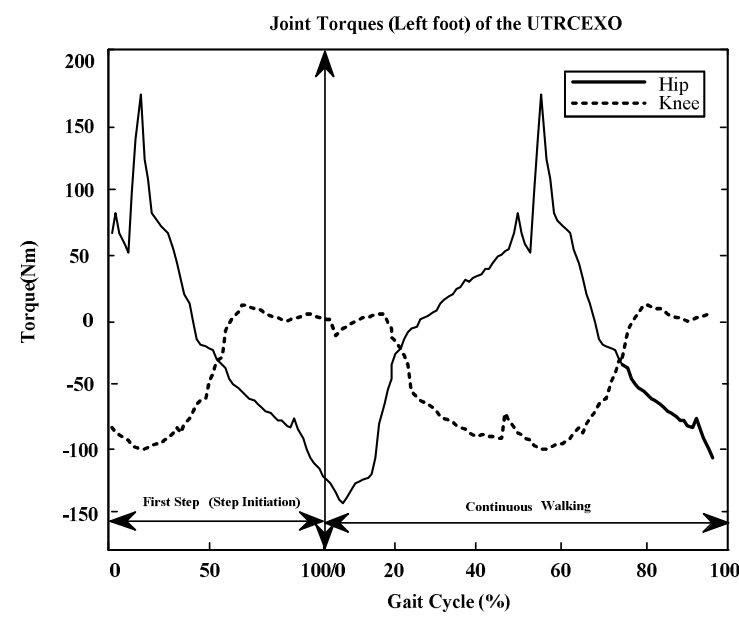

Fig. 8. Joint torques of the UTRCEXO for left foot

Fig. 7 and Fig. 8 show the joint torques for the hip and knee transmitted to the motion controller. As was mentioned in Section II, these joint torques were calculated from an inverse dynamics model based on human gait analysis. After step initiation, the UTRCEXO walked continuously with transmitted joint torques from $0 \%$ to $100 \%$ in the gait cycle during continuous walking. The UTRCEXO terminated its step according to the step termination intention of the operator using the same detection algorithm of walking intention. Fig. 9 and Fig. 10 show joint ROMs

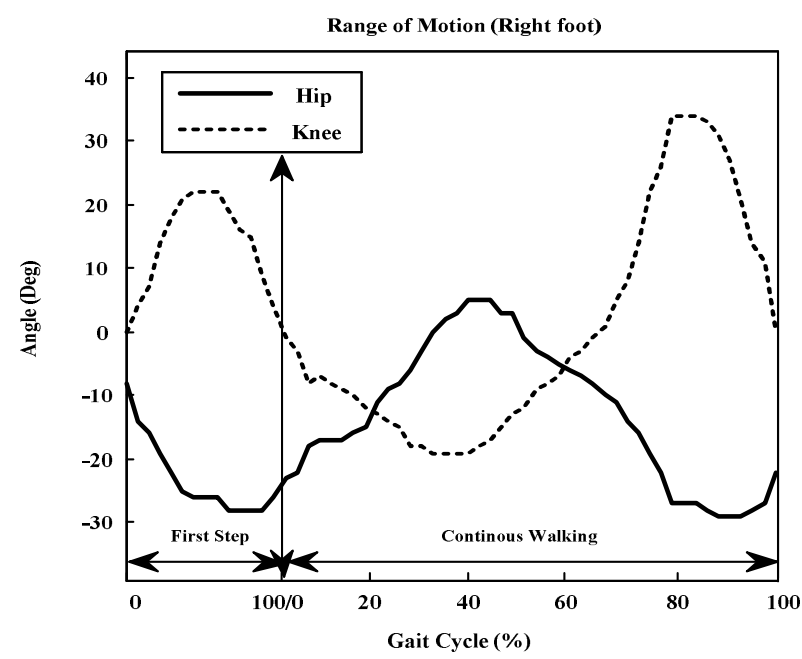

Fig. 9. Joint ROM (right foot) of the UTRCEXO

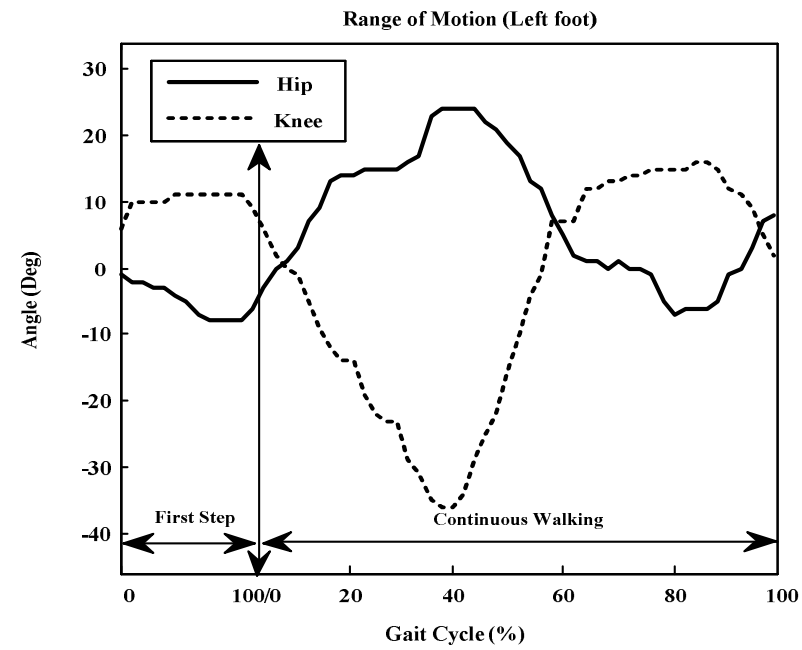

Fig. 10. Joint ROM (left foot) of the UTRCEXO

of the UTRCEXO for hip and knee. Joint ROMs of the UTRCEXO were similar to the human gait pattern analyzed in the previous study [25]. In that previous study, the joint ROMs were 0 to 60 degrees for the knee flexion and extension and 5 to 40 degrees for the hip flexion and extension. Table 2 shows a comparison of the ROM for each joint between the UTRCEXO and the human gait pattern analysis. Fig. 11 shows a comparison of the interaction forces between the UTRCEXO implementing the precedence walking assistance mechanism and the transparent UTRCEXO for the swing phase. In the transparent UTRCEXO, the operator equipped with the UTRCEXO walked, including step initiation, without any transmitted torques. In Fig. 11, the interaction forces indicate the pull force of the operator during the swing phase. The interaction forces between the transparent UTRCEXO and the operator were 0 to $96 \mathrm{~N}$, while the interaction forces between the UTRCEXO implementing the precedence walking assistance mechanism and the operator were 0 to $49 \mathrm{~N}$ in the swing phase. This clearly 
Table 2. Comparison of ROM between the UTRCEXO and the human gait pattern

\begin{tabular}{c|c|c}
\hline (Degree) & UTRCEXO & Human gait pattern \\
\hline Hip & $10 \sim 35$ & $5 \sim 40$ \\
\hline Knee & $0 \sim 55$ & $0 \sim 60$ \\
\hline
\end{tabular}

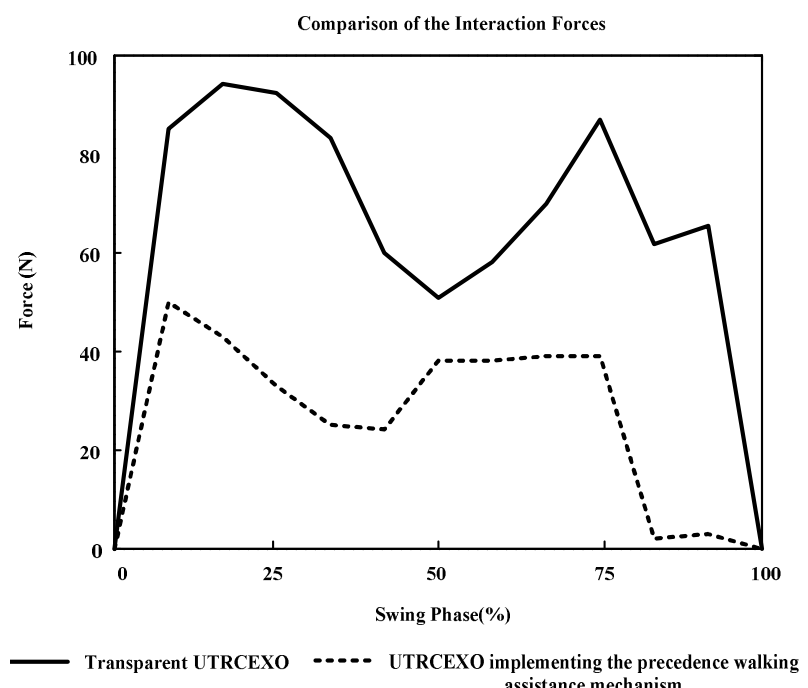

Fig. 11. Comparison result of the interaction forces

indicates that the UTRCEXO implementing the precedence walking assistance mechanism reduces the interaction force, which means that the operator can walk more comfortably even with a load.

\section{Discussion}

In this paper, we presented the walking assistive exoskeleton called the UTRCEXO, which implements the precedence walking assistance mechanism using bio-signals. We evaluated the UTRCEXO and demonstrated that it is currently capable of providing a reduction of interaction forces during the swing phase with a load.

The UTRCEXO detects the walking intention of the operator including step initiation using by analyzing the feedback from insole-type FSRs and F/T sensors, using an algorithm we developed based on MARB. We also developed a whole-body-type actuator for the UTRCEXO's joints, including a reduction gear, a motor, a sensor, and a controller. The UTRCEXO not only detects step initiation of the operator with the peak time approach using the insoletype FSRs prior to movement, but also walks continuously with reference joint torques. Additionally, by implementing the precedence walking assistance mechanism, the UTRCEXO can reduce the interaction forces during the swing phase, and also allow the operator to easily walk with a $10 \mathrm{~kg}$ load.

However, we implemented the precedence walking assistance mechanism in the UTRCEXO with reference joint torques calculated from an inverse dynamics model. It does not take the operator's desired step velocity into account. Additionally, even though there was a reduction of the interaction forces between the UTRCEXO and the operator, further reduction would be desirable.

As a result, future research with the UTRCEXO will focus on three areas. First, we will work to incorporate the intention of the operator with regards to step velocity into the precedence walking assistance mechanism during continuous walking. Second, we will attempt to further reduce the interaction forces incurred during the swing phase. Third, we will measure muscle activity during walking using EMG sensors to quantifiably evaluate how comfortable the UTRCEXO operator is when walking with a load.

\section{Acknowledgements}

This work was supported by the UTRC.

\section{References}

[1] A. M. Dollar, and H. Herr, "Lower Extremity Exoskeletonsand Active Orthoses; Challenges and State-of-the-Art," IEEE Transaction on ROBOTICS, 24 (1), pp. 144-158, 2008.

[2] J. L. Pons, "Wearable Robots: Biomechatronics exoskeletons," Wiley, pp. 165-198, 2008.

[3] H. Kazerooni, "Human power extender: an example of human-machine interaction via the transfer of power and information signal," in Proc. Int. Workshop on Advanced Motion Control, pp. 565-572, 1998.

[4] J. C. Moreno, E. Rocon, A. Ruiz, F. Brunetti, and J. L. Pons, "Design and implementation of an inertial measurement unit for control of artificial limbs: application on leg orthoses," Sensors and Actuators B, 118 (1-2, pp. 333-337), 2006.

[5] A. M. Dollar, and H. Herr, "Active orthoses for the Lower-Limbs: Challenges and State-of-the-Art," in Proc. Conf. Rehab. Robot, pp. 968-977, 2007.

[6] H. Kazerooni, J. -L. Racine, H. Lihua, and R. Steger, "On the control of the Berkeley Lower Extremity Exoskeleton (BLEEX)," in Proc. IEEE Int. Conf. Robot, pp. 4353-4360, 2005.

[7] P. Neuhaus, and H. Kazerooni, "Industrial-Strength Human-Assisted Walking Robots," IEEE ROBOT AUTOM MAG, 8(4), pp. 18-25), 2001.

[8] S. Mohammed, Y. Amirat, and H. Rifai, "Lower limb movement assistance through wearable robots: State of the Art and Challenges," Adv. Robotics26, pp. 1-22, 2012.

[9] D. Cha, S. N. Oh, K. I. Kim, K.-S. Kim and S. Kim, 
"Precedence walking assistance mechanism for exoskeletons with improved detection of step initiation based on gait analysis," Journal of Mechanical Science and Technology, To be published.

[10] T. Kawabata, H. Satoh and Y. Sankai, "Working posture control of robot suit HAL for reducing structural stress," in Proc. Int. Conf. on Robotics and Biomimetics, pp. 2013-2018, 2009.

[11] K. Suzuki, G. Mito, H. Kawamoto, Y. Hasegawa and Y. Sankai, "Intention-based walking support for paraplegia patients with robot suit HAL," Adv. Robotics 21, pp. 1441-1469, 2007.

[12] A. Tsukahara, R, Kawanish, Y. Hasegawa and Y. Sankai, "Sit-to-stand and stand-to-sit transfer support for complete paraplegic patients with robot suit HAL, Adv. Robotics 24, pp. 1615-1638, 2010.

[13] J. E. Pratt, B. T. Krupp, C. J. Morse, and S. H. Collins, "The RoboKnee: An Exoskeleton for Enhancing Strength and En-durance During Walking," in Proc. IEEE Int. Conf. Robot, pp. 2430-2435, 2004.

[14] R. Jimenez-Fabian, and O. Verlinden, "Review of control algorithm for robotic ankle systems in lower limb orthoses, prostheses," Medical Engineering \& Amp; Physics, 2011.

[15] A. B. Zoss, H. Kazerooni, and A. Chu, "Biomechanical Design of the Berkeley Lower Extremity Exoskeleton (BLEEX)," IEEE Trans. Mechatronics, 11(2), pp. 128-139), 2006.

[16] A. Chu, A. B. Zoss, and H. Kazerooni, "On the Biomimetic Design of the Berkeley Lower Extremity Exoskeleton (BLEEX)," in Proc. IEEE Int. Conf. Robot, pp. 4345-4352, 2005.

[17] J. Ghan, R. Steger, and H. Kazwerooni, "Control and system identification for the Berkeley lower extremity exoskeleton (BLEEX),"Adv. Robotics 20, pp. 9891014, 2006.

[18] H. Herr, "Exoskeletons and Orthoses: Classification, Design Challenges and Future Directions," J. Neuroeng Rehabil. Vol. 6, 2009.

[19] K. Kong, and D. Jeon, "Design and control of an exoskeleton for the elderly and patients," IEEE/ ASME Trans. Mechatron, 11, pp. 428-432, 2006.

[20] E. Alberto, T. Mukul, P. Andrew, and S. Michael, "The ReWalk Powered Exoskeleton to Restore Ambulatory Function to Individuals with ThoracicLevel Motor-Complete Spinal Cord Injury," American journal of physical medicine \& rehabilitation, vol. 91, pp. 911-921, 2012

[21] Gancet J, Ilzkovitz M, Cheron G, Ivanenko Y, van der Kooij H, van der Helm F, Zanow F, Thorsteinsson F. Mindwalker, "A brain controlled lower limbs exoskeleton for rehabilitation. Potential applications to space." 11th Symposium on Advanced Space Technologies in Robotics and Automation, 2011.

[22] L. Ren, R. K. Jones, and D. Howard, Whole body inverse dynamics over a complete gait cycle based only on measured kinematics, Journal of Biomechanics, 41 (12, pp. 2750-2759), 2008.

[23] D. Cha, H. T. Seo, S. N. Oh, K. I. Kim, K. -S, Kim, and S. Kim, "Verification of the peak time approach for detection of step initiation using the UTRCEXO," International journal of control, automations and systems, 12(5), pp. 1070-1076, 2014.

[24] D. Cha,S. N. Oh, K. I. Kim, K. -S. Kim, and S. Kim, "Implementation of the precedence walking assistance mechanism in an exoskeleton with only vertical ground reaction forces," Electron Lett, 50(3), pp. 146-148, 2014.

[25] D. Cha, D. Kang, K. I. Kim, K. -S. Kim, B. Lee, and S. Kim, "Faster detection of step initiation for the lower limb exoskeleton with vertical GRF events," Journal of Electrical Engineering and Technologies, 9(2), pp. 733-738, 2014.

[26] J. Jung, I. Jang, R. Riener, and H. Park, "Walking intention detection algorithm for paraplegic patients using a robotic exoskeleton walking assistant with crutches," International journal of control, automation, and systems, pp. 954-962, 2012.

[27] K. Lukas, M. Sanaz, and S. Harmut, "Decentralized evolution of robotic behavior using finite state machine," International journal of intelligent computing cybernetics, pp. 1-33, 2009.

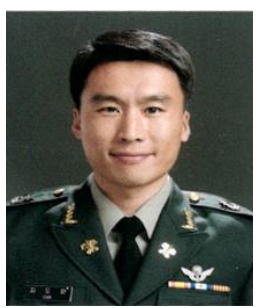

Dowan Cha received the B.A. degree in Management from Korea Military Academy in 2002. He received the MSc. degree in Computer Science from the University of Wales, UK in 2006 and another MSc. Degree in Artificial Intelligence from the University of Wales, UK in 2007. He received the Ph.D. degree in Mechanical Engineering from KAIST in 2014. His research interests include military robots, exoskeletons, the detection of human movement intention, biomechanics, human-robot interface and learning algorithm. He received the Best Session Paper Award from International Conference (ICMERA 2012) held in Romania in 2012, the Best Achievement Award from Brain Korea (BK)21 in 2013, and Young Scientist Award from Korea Robot Society(KROS) in 2014.

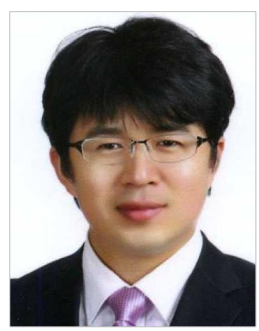

Sung Nam Oh received B.E. and M.E. degrees in Electrical Engineering from Myong Ji University in 2002, and 2004, respectively. He is currently working toward his Ph.D. at Myong Ji University. His research interests include exoskeletons, and humanoid robots. 


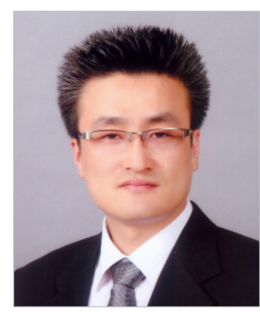

Hee Hwan Lee received B.S. in Mechatronics Engineering from Korea Polytechnic University in 2005. He is currently working towards his Ph.D at Myong Ji University. His research interests include exoskeleton, and embedded system.

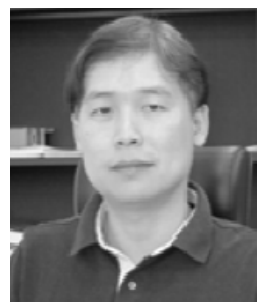

Kyung-Soo Kim received the B.S., M.S., and Ph.D. degrees in Mechanical Engineering from KAIST in 1993, 1995, and 1999, respectively. He was Chief Researcher with LG Electronics, Inc., from 1999 to 2003 and a DVD Group Manager with STMi croelectronics Company Ltd., from 2003 to 2005. In 2005, he joined the Department of Mechanical Engineering, Korea Polytechnic University, as a Faculty Member. Since 2007, he has been with the Department of Mechanical Engineering, KAIST. He serves as Associate Editors of Automatica and Journal of Mechanical Science and Technology. His research interests include the control theory, sensor and actuator design and robot manipulator design.

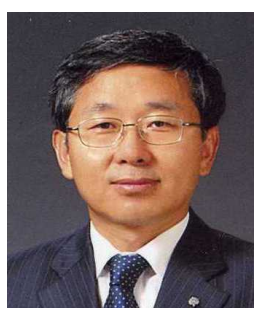

Kab Il Kim received the B.S. degree in Electrical Engineering from Seoul National University in 1979 . He received the M.S. degree in Electrical Engineering from KAIST in 1981 and Ph.D. Degree in Electrical Engineering from Clemson University in 1990. He was a full time instructor in Korea Military Academy form 1981 to 1985, and now is currently working in Myong Ji University since 1991. He was a research fellow of the Ohio State University in 1997, and Tsinghwa University from 1996 to 2003. His research interests include humanoids, exoskeletons, and control of robots. He received the Merit Award in 2011 and YHS Academic Award in 2012 form Korea Institute of Electrical Engineering (KIEE).

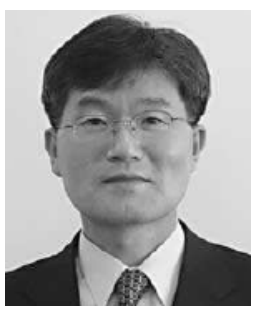

Soohyun Kim received the B.S. degree from Seoul National University in 1978, the M.S. degree from KAIST in 1980. He received the Ph.D. degree in 1991 form the Imperial College of Science, Technology and Medicine, University of London, the UK, all in Mechanical Engineering. He worked for Korea Military Academy as a Senior Lecturer at the Department of Ordnance Engineering from 1980 to 1984 , and for the Korea Institute of Technology at the School of Mechanical Engineering from 1984 to 1988. After his Ph.D., he joined the Faculty of the Department of Mechanical Engineering at KAIST in 1991. His research interests include robots, micro/nano actuator and sensor, and manipulator. 